\title{
FERMION DYNAMICS IN THE KALUZA-KLEIN MONOPOLE GEOMETRY
}

\author{
F Alexander BAIS \\ Lorentz Insttute for Theoretical Physics, Nieuwsteeg 18, 2311 SB Leiden, The Netherlands \\ Peter BATENBURG
}

Institute for Theoretical Physics, Princetonplein 5, PO Box 80 006, 3508 TA Utrecht, The Netherlands

Received 12 March 1984

\begin{abstract}
The behaviour of charged particles in the Kaluza-Klein monopole geometry is studied A discussion of the five-dımensional geodesics is followed by an analysis of the corresponding Dirac equation A main observation is that a charged particle cannot reach the core of the pole, in contrast with the conventional case For the Dirac particle this comes about because the dimensional reduction generates a radius dependent anomalous magnetıc moment which asymptotically yields a gyromagnetic ratio $g=1$ This has the following consequences (1) there is no Lipkin-WeisbergerPeshkın problem in the lowest angular momentum channel, (11) there are no bound states, (111) scattering leads to helicity-flip rather than to charge-exchange which would be another logical possibility Our results indicate that there is no phenomenon, analogous to the Rubakov-Callan effect to be expected for the Kaluza-Kleın monopole
\end{abstract}

\section{Introduction}

It is well known that finite-action (instanton) solutions of a field theory in $d$ euclidean dimensions, correspond to finite-energy (soliton) solutions if interpreted in $(d+1)$ dimensions This idea can be successfully applied to the gravitational instantons [1] to obtain solitons [2] (for an introduction to the subject see ref. [8]) in the original five-dımensional Kaluza-Kleın (KK) theory [3], whıch examplifies a geometrical unification of electromagnetism and gravity The metric may generically be written as

$$
\mathrm{d} s^{2}=g_{\mu \nu} \mathrm{d} x^{\mu} \mathrm{d} x^{\nu}+C\left(\mathrm{~d} \alpha+A_{\mu} \mathrm{d} x^{\mu}\right)^{2},
$$

where $g_{\mu \nu}$ is the four-dimensional metric, $C=C\left(x^{\mu}\right)$ some scalar function and $A_{\mu}$ the electromagnetic gauge potential (we have absorbed the fundamental charge into the definition of $A_{\mu}$ ) The philosophy is that the five-dimensional space factorizes locally into the four-dimensional physical spacetıme (with coordinates $x^{\mu}$ ) and some one-dimensional compact space (with coordinate $\alpha$ ). It is the compactness of the internal space ( 1 e the periodicity of $\alpha$ ) which effects the quantization of charge. In this paper we focus on the simplest instanton, the Taub-NUT solution [1], which in the Kaluza-Klein framework corresponds to a magnetıc monopole [2] Thus it appears that magnetic monopoles are unavoidable ingredients of any theory where 
electric charge is quantized intrinsically as is the case in both GUTs [4] and KK theories [2] This suggests that monopoles are not only a sufficient condition for charge quantization as was Dirac's key observation [5], but also a necessary condition

In this paper we shall consider the interactions of charged particles with the Kaluza-Klein monopole, and compare it where possible with the conventional situation for Dirac and 't Hooft-Polyakov monopoles It is found that several distınctive features arıse

(1) The classical pole-charge dynamics does not violate angular momentum conservation because even in the $J=0$ channel the charge can never reach the monopole core

(11) The dimensional reduction of the relevant Dirac equation, yields an $r$-dependent anomalous magnetic moment term which asymptotically tends to the value $\kappa=-\frac{1}{2}(1$ e a gyromagnetic ratio of $g=1)$

(111) The behaviour of the spinor wave function at the origin is such that the Lipkın-Weisberger-Peshkın problem [6] is evaded, without imposing a special boundary condition linking different charge components of the effective fourdimensional Dirac field In other words the scattering in the lowest allowed angular momentum channel is (like in the conventional abelian case) pure helicity-flip and does not lead to charge-exchange (as in the 't Hooft-Polyakov case) (for a review of charge-pole dynamics see, for example, [8]) This indicates that there will be no spectacular physıcs like a Rubakov-Callan effect [9] for the Kaluza-Kleın monopole, at least in the minimal $d=5$ model we consider

(1v) In the lowest angular momentum channel, there are no bound states of a charged particle to the Kaluza-Klein monopole The same is true in the conventional (abelian) case whenever $\kappa<0$ [10]

In the next section we consider the classical dynamics, 1 e we study the fivedimensional geodesics In sect 3 we discuss the Dirac equation Many of the results presented here have been announced in a previous publication [8]

\section{Geodesics - classical dynamics}

The simplest KK monopole corresponds to the Taub-NUT metric in the four spatial dimensions, whereas the time dimension is added trivially

$$
\mathrm{d} s^{2}=-\mathrm{d} t^{2}+A(r) \mathrm{d} r^{2}+r^{2}\left(\mathrm{~d} \theta^{2}+\sin ^{2} \theta \mathrm{d} \phi^{2}\right)+C(r)\left(\mathrm{d} \alpha+\frac{1}{2} \cos \theta \mathrm{d} \phi^{2}\right),
$$

with

where

$$
A=\left(1+\frac{m}{\rho}\right)^{2}, \quad C=\left(\frac{4 m r}{\rho+m}\right)^{2}
$$

$$
\rho^{2}=r^{2}+m^{2}
$$


For the solution to be nonsingular at $r=0$ the angle $\alpha$, which parametrizes the internal space $S^{1}$, should have periodicity $2 \pi$ This value of the period is also necessary to satisfy the Dirac quantization condition of the magnetic charge In fact comparing ( 11 ) and ( 21 ) one obtains that $\boldsymbol{A}_{\mu}$ describes a monopole field of strength eg/ $4 \pi=-\frac{1}{2}$ We note that the choice of the radial coordinate was made so as to obtain the standard form for the angular $(\theta, \phi)$ part of the metric The parameter $m$ which appears in (21) is the so-called Taub-NUT parameter, which should be positive to avoid singularities and sets the scale for the internal radius

$$
R_{\infty}=\sqrt{C(\infty)}=4 m,
$$

which in its turn can be expressed in terms of the physical fine-structure constant $\alpha$ and the gravitational constant $G$ as

$$
R_{\infty}=2 \sqrt{G / \alpha} \sim 10^{-32} \mathrm{~cm}
$$

A lagrangian density for a unit-mass test particle is given by

$$
\begin{aligned}
\mathscr{L} & =\frac{1}{2} \gamma_{\Sigma} x^{\Sigma} x^{1} \\
& =\frac{1}{2}\left[-\dot{i}^{2}+A r^{2}+r^{2}\left(\theta^{2}+\sin ^{2} \theta \phi^{2}\right)+C\left(\alpha+\frac{1}{2} \cos \theta \phi\right)^{2}\right],
\end{aligned}
$$

where the dot refers to differentiation with respect to proper time

There are three conserved quantities associated with the Killing vectors $\partial_{t}, \partial_{\phi}$ and $\partial_{\alpha}$

$$
\begin{aligned}
& p_{t}=-t \equiv-E, \\
& p_{\alpha}=C\left(\alpha+\frac{1}{2} \cos \theta \dot{\phi}\right) \equiv-2 S, \\
& p_{\phi}=r^{2} \sin ^{2} \theta \phi-S \cos \theta .
\end{aligned}
$$

From these one may construct a conserved angular momentum

$$
\boldsymbol{J}=\boldsymbol{L}-\boldsymbol{S} \hat{\boldsymbol{r}},
$$

where $\boldsymbol{L}$ is the standard orbital part

$$
\left.\boldsymbol{L}=r^{2}(\theta \hat{\boldsymbol{\phi}}-\sin \theta \phi \hat{\boldsymbol{\theta}})\right),
$$

and the radial part $-S \hat{r}$ corresponds to the angular momentum $(-q g \hat{r} / 4 \pi)$ of a charge $q$ in the field of a pole with strength $g$ [8]. The fact that both $L$ and $S$ are conserved fixes the motion of the particle to lie on a cone with opening angle $2 \beta$ where

$$
\beta=\operatorname{arctg}(|\boldsymbol{L}| / S)
$$

The radial motion is determined by the constants $L, S$ and $E$, and most easily obtained from the energy-momentum relation

$$
\gamma^{1 \Sigma} p_{1} p_{\Sigma}=-1
$$


This yields the equation

$$
A r^{2}+\frac{L^{2}}{r^{2}}+(2 S)^{2}\left(\frac{1}{R(r)^{2}}-\frac{1}{R_{\propto}^{2}}\right)=E^{2}-\left(1+\frac{(2 S)^{2}}{R_{x}^{2}}\right)
$$

Note that the effective four-dımensional rest mass

$$
M_{0}^{2}=1+(2 S)^{2} / R_{x}^{2},
$$

contains a contribution proportional to the fifth component of the momentum $p_{\alpha}$ which in turn is proportional to the charge of the particle The radial motion is now determined through the equation

$$
\frac{1}{2} A r^{2}+\frac{L^{2}}{2 r^{2}}+\frac{1}{2}(2 S)^{2}\left(\frac{1}{R^{2}}-\frac{1}{R_{x}^{2}}\right)=\frac{1}{2}\left(E^{2}-M_{0}^{2}\right),
$$

and may be compared with the motion of a particle with $r$-dependent mass $A$ and "energy" $\frac{1}{2}\left(E^{2}-M_{0}^{2}\right) \geqslant 0$ in a potential

$$
V(r)=\frac{L^{2}}{2 r^{2}}+\frac{1}{2}(2 S)^{2}\left(\frac{1}{R^{2}}-\frac{1}{R_{x}^{2}}\right)
$$

It is at this point that an important difference with the conventional problem occurs, because in that case the term proportional to $S^{2}$ is absent, leading to a well known pathology A radially infalling particle $(L=0)$ would not feel any (Lorentz) force and hence go straight through the monopole, en passant violating the conservation of total angular momentum $\boldsymbol{J}$ (because $\hat{\boldsymbol{r}}$ flips direction) This is the classical manıfestation of the Lipkın-Weisberger-Peshkın problem [6]

In the present case of the $\mathrm{KK}$ monopole the situation is much healthier indeed Even if $L=0$ a repulsive $1 / r^{2}$ core due to the term proportional to $S^{2}$ prevents the particle from reaching the origin and angular momentum conservation is saved

It is amusing to contemplate the $L=0$ dynamics in the five-dimensional setting We may set $\theta=\theta=\phi=0$ and are left with geodesics on the two-dimensional $r=z$, $x^{5}=\alpha$ space, which is a curved conical surface described by the metric

$$
\mathrm{d} s^{2}=-\mathrm{d} t^{2}+A \mathrm{~d} r^{2}+C \mathrm{~d} \alpha^{2}
$$

One may obtain an embedding of the spatial surface in $R^{3}$ by rotatıng the curve $\sqrt{C}=R$ around an axis $\sigma$ so that the metric takes the standard form

$$
\mathrm{d} s^{2}=\left[1+\left(\frac{\mathrm{d} R}{\mathrm{~d} \sigma}\right)^{2}\right] \mathrm{d} \sigma^{2}+R^{2} \mathrm{~d} \alpha^{2}
$$

Comparıng (2 17) and (2 16) one obtains

$$
\sigma(r)=2 m \int_{1}^{r} \sqrt{\frac{(1+y)\left(1+y^{2}\right)}{y^{3}}} \mathrm{~d} y
$$


where

$$
x=\frac{1}{2}\left(1+\sqrt{1+(r / m)^{2}}\right)
$$

Clearly for large $r$ we have that $\sigma(r) / r \rightarrow 1,1$ e the cone turns into a cylinder asymptotically An incoming geodesic spirals around the cone until the turning point is reached, at which point the geodesic starts spiralling out This so-called magnetic mirror effect is very similar to what happens in the conventional case if one considers the motion of a charged particle with $L \neq 0$

For the aficionados of classical mechanics there is one more amusing observation

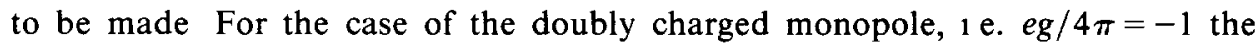
dynamical system admits an interpretation as a symmetric top [13]. We recall that the lagrangian for a symmetric top is [11]

$$
\mathscr{L}_{\mathrm{ST}}=\frac{1}{2} I_{1}\left(\theta^{2}+\sin ^{2} \theta \phi^{2}\right)+\frac{1}{2} I_{3}(\alpha+\cos \theta \phi)^{2}
$$

Apparently the geodesic motion in the monopole geometry contains that of a symmetric top with moments of inertia $I_{1}=r^{2}$ and $I_{3}=\left(\frac{1}{2} R\right)^{2}$ The free top performs two kinds of motion

(1) the precession around the $J$-axis with energy $J^{2} \sin ^{2} \beta / 2 I_{1}=L^{2} / 2 I_{1}$,

(ii) the rotation around its own axis with energy $J^{2} \cos ^{2} \beta / 2 I_{3}=S^{2} / 2 I_{3}$. Clearly the geodesic lagrangian adds a translational motion to this picture, as both the mass and the moments of inertia depend on the position $r$, energy is constantly converted from translational to rotational energy or vice versa

For completeness we recall briefly a remark made in ref [2], concerning the neutral test particle. From (2 14) it is obvious that a solution to the geodesic equations 1s provided by having $L=S=E^{2}-M_{0}^{2}=0$, and hence $r=0$ A neutral particle can apparently be at rest in the KK monopole geometry This is related to the surprising fact that the gravitational mass appearing in the newtonian potential vanishes for the $\mathrm{KK}$ monopole as follows immediately from the fact that $g_{00}=-1$

\section{Fermion dynamics}

This section is devoted to an analysis of the Dirac equation in the five-dimensional spacetıme corresponding to the Kaluza-Klein monopole. Our aim is to perform the dimensional reduction and compare the result with the conventional fourdimensional Dirac equation in the field of an abelian monopole. This latter problem has been studied repeatedly and most thoroughly by Goldhaber, Kazama and Yang $[7,10]$

The five-dımensıonal Dirac equatıon, covariant with respect to general coordinate and local Lorentz transformations reads

$$
D \tilde{\psi}=e_{C}^{\Sigma} \gamma^{C}\left(\partial_{\Sigma}+\omega_{\Sigma A B} \frac{1}{8}\left[\gamma^{A}, \gamma^{B}\right]\right) \tilde{\psi}=0,
$$

where $e^{\Sigma}$ and $\omega_{\Sigma A B}$ are the inverse frame-field (funfbein) and the spin connection 
respectively The five-dimensional gamma matrices $\gamma^{A}$ (flat indices) are just the usual $4 \times 4$ matrices satısfying

$$
\left\{\gamma^{4}, \gamma^{B}\right\}=2 \eta^{4 B}
$$

where $\eta^{A B}$ is the five-dimensional Minkowski metric The dimensional reduction of eq (3 1) is achieved by making a harmonic analysis of the spinor wave function on the internal space This amounts to decomposing $\tilde{\psi}$ as a Fourier series on $S^{1}$ with coordinate $\alpha(0 \leqslant \alpha<2 \pi)$

$$
\tilde{\psi}\left(x^{\mu}, \alpha\right)=\sum_{n=-x}^{\infty} \tilde{\psi}_{n}\left(x^{\mu}\right) \mathrm{e}^{i n \alpha}
$$

Indeed, the single five-dimensional equation is equivalent to an infinite set of uncoupled four-dimensional equations, which upon substitution of the general Kaluza-Klein metric ( 11 1) take the form

$$
\left\{\gamma^{\mu}\left(\partial_{\mu}-\ln A_{\mu}+\frac{1}{8}\left[\gamma^{a}, \gamma^{b}\right] \omega_{\mu a b}+\partial_{\mu} R / 2 R\right)+M-\frac{n}{|n|} \frac{1}{16} l\left[\gamma^{\mu}, \gamma^{\nu}\right] F_{\mu \nu} R\right\} \psi_{n}\left(x^{\lambda}\right)=0
$$

Here some remarks are in order

(1) The indices $\mu$ (curved) and $a$ (flat) run from 0 to $3 F_{\mu \nu}$ is the abelian field strength corresponding to $A_{\mu}$ We have introduced $C=R^{2}$ where $R(r)$ is the radius of the internal space $\mathrm{S}^{1}$

(11) The matices $\gamma^{\mu}$ with curved indices are defined in the four-dimensional context

$$
\gamma^{\mu} \equiv e_{a}^{\mu} \gamma^{a} \quad(a=0, \quad, 3)
$$

Thus in contrast with their five-dimensional analogs

$$
\Gamma^{\Sigma} \equiv e_{A}^{\Sigma} \gamma^{4}
$$

(111) The chirally rotated field $\psi_{n}$ is defined in terms of the original $\tilde{\psi}_{n}$ as

$$
\psi_{n}=\frac{1}{2}\left(1-\gamma_{5}\right) \tilde{\psi}_{n} \pm \frac{1}{2} \boldsymbol{l}\left(1+\gamma_{5}\right) \tilde{\psi}_{n}, \quad(n= \pm|n|)
$$

This transformation is applied to obtain the conventional positive mass term in (34) where

$$
M=\frac{|n|}{R}
$$

Note that the mass is $r$-dependent for the case we are interested in In view of the fact that the asymptotic value of $R$ is fixed by (25) one concludes that the mass scale is of the order of the Planck mass

(Iv) From the coupling to the photon field $A_{\mu}$ in (34) we verify that the charge $q$ of $\psi_{n}$ equals $q=n e$ as expected From $(38)$ we furthermore infer that the mass 
is proportional to the charge This implies that by finetuning the pseudoscalar mass term in the five-dimensıonal theory we can at most arrange two states of the infinite tower $\left\{\psi_{n}\right\}$, to be massless in $d=4$ For each low-mass particle $\left(M \ll M_{P}\right)$ in the $d=4$ theory we have to introduce a different field in the $d=5$ theory

(v) It is interestıng to note that the last term in eq (34) describes the effect of an anomalous magnetic moment, corresponding to the so-called "Paulı term" in the nonrelatıvistic theory Comparing the usual expression

$$
\Delta \mathscr{H}=\frac{l \kappa n}{M} F_{\mu \nu} \frac{1}{8}\left[\gamma^{\mu}, \gamma^{\nu}\right],
$$

with (3 4) we obtain that for the Kaluza-Kleın theory $\kappa$ has the particular value

$$
\kappa=-\frac{1}{2}
$$

In other words the gyromagnetıc ratio $g=2(1+\kappa)=1$

For the Kaluza-Klein monopole geometry we know $\gamma^{\mu}, \omega_{\mu a b}, F_{\mu \nu}$ and $A_{\mu}$ explicitly A natural choice for the frame is

$$
\tilde{e}^{0}=\mathrm{d} t, \quad \tilde{e}^{1}=\Lambda \mathrm{d} r, \quad \tilde{e}^{2}=r \mathrm{~d} \theta, \quad \tilde{e}^{3}=r \sin \theta \mathrm{d} \phi,
$$

where $A=\sqrt{A(r)}$

The (Levi-Civita) spin connection one-form is defined through

$$
\begin{gathered}
\mathrm{d} \tilde{\boldsymbol{e}}^{a}+\tilde{\boldsymbol{\omega}}_{b}{ }_{b} \wedge \tilde{\boldsymbol{e}}^{b}=0, \\
\tilde{\omega}_{a b}=-\tilde{\omega}_{b a}
\end{gathered}
$$

Using (3 11) one obtains

$$
\begin{aligned}
& \tilde{\omega}_{\theta 21}=1 / \Lambda, \\
& \tilde{\omega}_{\phi 31}=(\sin \theta) / \Lambda, \\
& \tilde{\omega}_{\phi 32}=\cos \theta,
\end{aligned}
$$

all other components vanish. However, in order to obtain an equation which allows a direct comparison with the usual four-dimensional equation in the field of an abelian monopole, it is convenient to make the following local Lorentz transformation

$$
e^{a}=\Omega^{a}{ }_{b} \tilde{e}^{b},
$$

with

$$
\Omega=\left(\begin{array}{cccc}
1 & 0 & 0 & 0 \\
0 & \sin \theta \cos \phi & \cos \theta \cos \phi & -\sin \phi \\
0 & \sin \theta \sin \phi & \cos \theta \sin \phi & \cos \phi \\
0 & \cos \theta & -\sin \theta & 0
\end{array}\right)
$$


This is of course just the transformation which would transform the frame (311) in the case of a flat spacetime ( $1 \mathrm{e} .1=1)$ into the cartesian frame $e^{a}=\mathrm{d} x^{a}$, with vanıshing spin connection Indeed the sole purpose of makıng the transformation here is exactly to get rid of the contribution of the angular components of the spin connection in the Dirac operator In the basis thus obtained the resulting equations read

$$
\begin{aligned}
& \left\{-\frac{1}{\Lambda}(\boldsymbol{\alpha} \cdot \hat{\boldsymbol{r}})\left(-\imath \partial_{r}+\frac{t}{2 r}(1-1)\right)+\frac{1}{r}(\boldsymbol{\alpha} \cdot \hat{\boldsymbol{\theta}})\left(-t \partial_{\theta}\right)\right. \\
& \left.\quad+\frac{1}{r \sin \theta}(\boldsymbol{\alpha} \cdot \hat{\boldsymbol{\phi}})\left(-t \partial_{\phi}-\frac{1}{2} n \cos \theta\right)+\beta M-\frac{1}{8} \frac{n}{|n|} \frac{R}{r^{2}} \beta(\boldsymbol{\sigma} \cdot \hat{\boldsymbol{r}})\right\} \psi_{n}=E \psi_{n}
\end{aligned}
$$

Here $\alpha$ and $\beta$ are the usual Dirac matrices The angular part of the equation is exactly the same as in the conventional case and the same analysis goes through [7] The conserved angular momentum is

$$
\boldsymbol{J}=\boldsymbol{r} \times \boldsymbol{\pi}-\mu \hat{\boldsymbol{r}}+\frac{1}{2} \boldsymbol{\sigma},
$$

where

$$
\mu=\frac{q g}{4 \pi}=-\frac{1}{2} n
$$

It is most convenient to simultaneously diagonalıze the operators $\mathscr{H}, J^{2}, J_{z}$ and $\boldsymbol{\sigma} \cdot \hat{\boldsymbol{r}}$ with eigenvalues $E, J(J+1), m$ and $s$ respectively From now on we will suppress the index $n$. We will also restrict ourselves to the most interesting mode which is the lowest angular momentum state with

$$
J=|\mu|-\frac{1}{2}
$$

This is the channel where peculiar things do happen in the cases of the Dirac as well as the 't Hooft-Polyakov monopoles A remarkable fact is that in this channel $(\boldsymbol{\sigma} \cdot \hat{\boldsymbol{r}})$ can only have a single eigenvalue $s=\mu /|\mu|$ Choosing the two-component elgenspinors $\eta_{m}$ accordingly

$$
(\boldsymbol{\sigma} \cdot \hat{\boldsymbol{r}}) \eta_{m}=\frac{\mu}{|\mu|} \eta_{m},
$$

we have

$$
\psi=\left(\begin{array}{l}
f(r) \eta_{m} \\
g(r) \eta_{m}
\end{array}\right)
$$

To find out what the operator $(\boldsymbol{\sigma} \cdot \pi)$ does on the spinors $\eta_{m}$ we exploit the useful identity

$$
\begin{aligned}
(\boldsymbol{\sigma} \cdot \hat{\boldsymbol{r}})(\boldsymbol{\sigma} \cdot \boldsymbol{\pi}) & =\hat{\boldsymbol{r}} \cdot \boldsymbol{\pi}+\boldsymbol{t \sigma} \cdot(\hat{\boldsymbol{r}} \times \boldsymbol{\pi}) \\
& =\hat{\boldsymbol{r}} \cdot \boldsymbol{\pi}+\frac{t}{r}\left(\boldsymbol{J}^{2}-L^{2}-\frac{3}{4}\right)+\frac{l}{r}|\boldsymbol{\mu}|
\end{aligned}
$$


In other words

$$
(\boldsymbol{\sigma} \cdot \pi) f \eta_{m}=-\imath \frac{\mu}{|\mu|}\left(\frac{1}{\Lambda} \partial_{r}+\frac{1}{2 r}+\frac{1}{2 \Lambda r}\right) f \eta_{m}
$$

Using this relation we arrive at the radial equations

$$
\begin{aligned}
& {\left[(E-M)-\frac{1}{8} \frac{R}{r^{2}}\right] f+\imath \frac{\mu}{|\mu|}\left(\frac{1}{\Lambda} \partial_{r}+\frac{1}{2 r}+\frac{1}{2 \Lambda r}\right) g=0,} \\
& {\left[(E+M)+\frac{1}{8} \frac{R}{r^{2}}\right] g+t \frac{\mu}{|\mu|}\left(\frac{1}{\Lambda} \partial_{r}+\frac{1}{2 r}+\frac{1}{2 \Lambda r}\right) f=0}
\end{aligned}
$$

These equations are identical to the conventional ones (with $\kappa=-\frac{1}{2}$ ) if $\Lambda=1$ and $R=R_{\infty}, 1$ e in the asymptotic region where $r \gg R_{\infty}$ This implies that the asymptotic behaviour of the solutions will be similar as well In the remainder of this section we first discuss the scattering solutions and then the bound-state problem.

\section{SCATTERING SOLUTIONS}

The scattering solutions for $j=|\mu|-\frac{1}{2}$ fermions coincide for $r \gg R_{\infty}$ with the solutions in the field of an abelian monopole, and are of the form [7]

$$
\psi\left(r \gg R_{\infty}\right) \sim\left(\begin{array}{c}
\frac{\mu}{|\mu|} \frac{1}{r} \sin (k r+\delta) \eta_{m} \\
\frac{1}{t r}\left(\frac{k}{E+M}\right) \cos (k r+\delta) \eta_{m}
\end{array}\right),
$$

where $k^{2}=E^{2}-M^{2}>0$ The phase shift $\delta$ will ultimately be fixed through the boundary condition at the origin. The small- $r$ behaviour of (3 23) differs essentially from the conventional case, due to the nontrivial radial dependence of $A$ and $R$ Defining

$$
G=\imath g, \quad F=-\frac{\mu}{|\mu|} f,
$$

one finds that the leading behaviour near $r=0$ is determined by

$$
\begin{aligned}
& \left(\partial_{r}+\frac{3}{2 r}\right) G+\frac{1}{r}\left(|n|+\frac{1}{2}\right) F=0, \\
& \left(\partial_{r}+\frac{3}{2 r}\right) F+\frac{1}{r}\left(|n|+\frac{1}{2}\right) G=0
\end{aligned}
$$

The approprate solution is the one where

$$
F(r=0)=-G(r=0) \sim r^{|n|-1} .
$$


Consequently the wave function $\psi$ does not vanish at the origin for the minimal case where $J=0\left(|\mu|=\frac{1}{2}\right)$ At first sight this seems to imply that we encounter the Lıpkın-Weısberger-Peshkın problem [6], being that the Jacobı 1dentity for the kınetıc momentum operators is not satisfied on the physical states This problem is a consequence of the fact that in the presence of a singular monopole

$$
\frac{1}{2} \varepsilon_{\eta j}\left[\pi_{1},\left[\pi_{j}, \pi_{k}\right]\right]=e \boldsymbol{\nabla} \cdot \boldsymbol{B}=\mu \delta^{3}(\boldsymbol{r})
$$

In the four-dimensional theory there are several ways out of this problem

(1) the addition of an anomalous magnetıc moment term à la Kazama, Yang and Goldhaber [7], which even if it is infinitesimally small, forces the wave function to vanısh at the origin,

(11) one may have a cancellation of contributions to (328), as is the case in the abelian limit of the 't Hooft-Polyakov monopole, where integrability at $r=0$ forces charge-exchange to occur [8]

It is interesting to note that the second possibility is conceivable in the present context, where we do have two degenerate components with opposite charge $\left(\psi_{|n|}\right.$ and $\psi_{-|n|}$ ) The resolution is however rather trivial, basically because the condition on $\psi(0)$ is less stringent The volume element contains an extra factor $R(r)$ which for small $r$ behaves like $R(r \rightarrow 0) \simeq 2 r$ Consequently matrix elements of (3 28) will always vanısh if

$$
\psi(r \rightarrow 0) \sim r^{-1 / 2+|\varepsilon|},
$$

with $\varepsilon$ arbitrary This condition is met for all $n$ (and all $J$ ), hence the LWP problem does in fact not occur The point is basically that the effective four-dimensional wave function $\psi_{4}=\sqrt{2 \pi R} \psi_{5}$ does vanish at $r=0$ We should not be too surprised with this result in view of the fact that also at the classical level the problem of a charged particle moving through the monopole core does not occur as we pointed out in the previous section

The existence of a solution which is well behaved at the origin and tends to the solution (3 24) for large $r$ implies that the scattering amplitude in the minımal angular momentum channel describes pure helicity-flip This is not so surprising either, the natural presence of the anomalous magnetıc moment term in (34) breaks helicity conservation explicitly This in turn is related to the fact that in the massless five-dimensional Dirac equation there is in general no chiral symmetry

\section{BOUND STATES}

We now investigate whether the system ( 323 ) possesses any bound states It is easy to verify that the appropriate boundary condition guaranteeing integrability at infinity is given by

$$
F(r \rightarrow \infty) / G(r \rightarrow \infty)=+\sqrt{\frac{M_{\infty}+E}{M_{\propto}-E}}
$$


Both $F$ and $G$ decay exponentially as $\exp (-k r)$ with $k^{2}=M_{\infty}^{2}-E^{2}$ For small $r$ the behaviour is the same as discussed before and determined by (326) as

$$
F(r \rightarrow 0) / G(r \rightarrow 0)=-1
$$

The question is whether the equations admit solutions (with $0 \leqslant|E|<M_{\infty}$ ) which interpolate between ( 331 ) and (3 30) To decide on this question it is convenient to consider the equation for the variable

$$
v=F / G
$$

which reads

$$
\frac{\mathrm{d} v}{\mathrm{~d} r}=-\Lambda\left(M+E+R / 8 r^{2}\right)+\Lambda v^{2}\left(M-E+R / 8 r^{2}\right)
$$

It is evident that the right-hand side vanishes for

$$
v_{ \pm}(r)= \pm \sqrt{\frac{M+E+R / 8 r^{2}}{M-E+R / 8 r^{2}}}
$$

In fig. 1 we have depicted the curves (3 34) for $E>0$. We also indicated the fact that $\mathrm{d} v / \mathrm{d} r$ is positive for $v>\left|v_{ \pm}\right|$and negative for $v<\left|v_{ \pm}\right|$by arrows pointing upward and downward respectively It is then clear that the solution which starts off at the origin with the correct behaviour $v(0)=-1$ as in (3 31), is bound to stay in the shaded region $(v-(r) \leqslant v(r) \leqslant-1)$ for all $r$ Hence it cannot have the correct behaviour at infinity For the case $E=0$, we have the exact solution $v(r)=-1$, which has the same problem of connecting with the wrong solution at $r=\infty$ The same argument goes through for $E<0$, and we conclude that there exist no bound-state solutions This situation may be compared to the conventional case where also no bound states exist in the lowest angular momentum channel if the anomalous magnetic moment is negative $(\kappa<0)$ The non-existence of fermionic zero modes

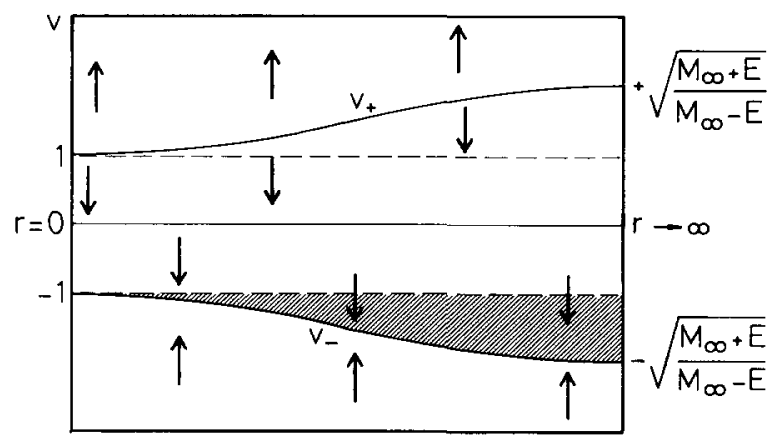

Fig 1 Plot of the functions $v_{ \pm}(r)$ for which $\mathrm{d} v(r) / \mathrm{d} r=0$ If $v(r)<\left|v_{ \pm}(r)\right|$ then $\mathrm{d} v / \mathrm{d} r<0$ The solution $v(r)$ which satisfies $v(0)=-1$ remains in the shaded area for all $r$ 
$\left(J=|\mu|-\frac{1}{2}\right)$ may be interpreted as the absence of fermionıc spın degrees of freedom for the Kaluza-Klein monopole, this in contrast with the 't Hooft-Polyakov-type monopoles in the presence of fermions [12], which do have zero modes

\section{Conclusions}

We have analysed the dynamics of charged classical - as well as Dirac - partıcles in the Kaluza-Klein monopole geometry The results are simple and deviate from the conventional picture for an abelian (or nonabelian) monopole The most outstanding difference is that a charged particle cannot reach the core of the monopole (which is basically due to the natural presence of an anomalous magnetic moment in the case of the fermions) The scattering of Dirac particles is pure helicity-flip and though conceivable, no charge exchange does occur There are no bound-state solutions Our results indicate that the "complete" fermion dynamics, where the back-reaction of the monopole (geometry) is taken properly into account, will not exhibit such spectacular phenomena as grand unified monopoles do It appears that there is no gravitational analog of the Rubakov-Callan effect, certainly in the minımal model we have considered

\section{References}

[1] T Eguchı, P B Gilkey and A J Hanson, Phys Rev 66 (1980) 215

[2] R D Sorkın, Phys Rev Lett 51 (1983) 87.

D J Gross and M J Perry, Nucl Phys B226 (1983) 29

[3] Th Kaluza, Sitzungsber Preuss Akad Wiss, Berlın, Math Phys KI (1921) 966 , O Klein, Z Phys 37 (1926) 895

[4] G 't Hooft, Nucl Phys B79 (1974) 276, A M Polyakov, JETP Lett 20 (1974) 194

[5] P A M Dirac, Proc Roy Soc, Series A 133 (1931) 60

[6] H J Lipkin, W I Weisberger and M Peshkın, Ann of Phys 53 (1969) 203

[7] Y Kazama, C N Yang and A S Goldhaber, Phys Rev D15 (1977) 2287

[8] F A Bats, Lectures presented at the summer institute, Progress in gauge field theory, Cargèse, Sept $1-151983$

[9] V A Rubakov, JETP Lett 33 (1981) 644, Nucl Phys B203 (1982) 311 , C G Callan, Phys Rev D25 (1982) 2141, D26 (1982) 2058, Nucl Phys B212 (1983) 391

[10] Y Kazama and C N Yang, Phys Rev D15 (1977) 2300

[11] L D Landau and E M Lifshitz, Mechanics (Pergamon, 1960)

[12] R Jackiw and C Rebbl, Phys Rev D13 (1976) 3398,

E Witten and D Olive, Phys Lett 78B (1978) 99

[13] M Fierz, Helv Phys Acta 17 (1944) 27 